\section{Towards a General Understanding of Bacterial Interactions}

\author{
Zhijie Zhang, ${ }^{1}$ \\ Mark van Kleunen, ${ }^{1,2}$ \\ Lutz Becks, ${ }^{3}$ and \\ Madhav P. Thakur (1), 1,
}

Understanding the general rules of microbial interactions is central for advancing microbial ecology. Recent studies show that interaction range, interaction strength, and community context determine bacterial interactions and the coexistence and evolution of bacteria. We highlight how these factors could contribute to a general understanding of bacterial interactions.

\section{Microbial Community Ecology}

A central tenet of community ecology is to understand the rules of species interactions and their consequences for community structure, such as species diversity. Historically, microbial interactions and their outcomes were studied to test theories of community ecology (e.g., $[1,2])$ which were developed for plants and animals $[3,4]$. This trend has changed remarkably in the last few years with rapid progress in microbial community ecology due mainly to the continued growth of molecular technologies $[4,5]$. Today, microbial community ecology has become an integral branch of ecology and microbiology and it continues to advance our understanding of the implications of microbial interactions for disease control, food production, climate change mitigation, and biodiversity conservation.

Bacteria are among the most studied microorganisms in microbial community ecology, particularly when it comes to experimental assessments of microbial interactions [4]. Yet, we still lack a consensus for how bacterial species interact, and how those interactions may regulate the diversity of bacteria [6]. This is due to several key challenges in studying bacterial interactions, such as delineating the spatial and temporal scale at which bacteria inter act, quantifying the strength of their interactions, dependence of their interactions on abiotic conditions, and constantly varying interactions owing to rapid evolution and changes in bacterial abundance. Three recent studies provide insight into overcoming these challenges [7-9]. These studies independently show interaction range [7], interaction strength [8] and community context [9] as three factors determining bacterial species interaction and the coexistence and evolution of bacteria. We highlight how simultaneous consideration of these three factors can help to achieve a more general understanding of bacterial interactions (Figure 1) and their relevance for building predictive models for microbial community ecology.

\section{Interaction Range, Interaction} Strength, and Community Context Interaction range is the spatial domain in which an individual can interact with other individuals of either the same species or a different species. Usually, the interaction ranges of several bacterial cells are limited to their close surroundings, such as biofilms [10]. However, even within biofilms, some cells are close neighbors while others are distant. Whether bacteria cells in spatially structured biofilms interac with close and distant neighbors is not well understood given the complexity in empirically quantifying the distances between bacteria during their interactions. Dal Co et al. [7] used an elegant approach of measuring the interaction range of bacterial cells as the size of the neighborhood from which one bacterial cell can retrieve the amino acids produced by neighboring cells. They highlight that the interaction range of bacteria is short, that is, individuals interact only with their immediate neighbors. More specifically, they used two genotypes of Escherichia coli that exchange essential amino acids for their growth. They showed that the growth of one genotype was promoted when most of its neighbors in its interaction range were of the other genotype [7] This study contributes to a general understanding of how the range of interaction matters for facilitation and coexistence at the cellular level.

While the size of the interaction range of a bacterial cell indicates the distance over which it can affect another cell (Figure 1), the direction (mutualistic or antagonistic) of these bacterial interactions is likely to depend on how bacterial cells modify their immediate environment [11]. Towards this end, Ratzke et al. [8] showed that increased nutrient availability promoted the ability of soil bacterial species to modify their environment (e.g., pH values), which subsequently magnified the negative interactions between soil bacterial species. Interestingly, this study highlighted that the strength of negative interactions between bacterial species was mediated mainly by the production of toxic metabolites and could not be attenuated by refreshing nutrients. These strong competitive interactions between bacteria species impeded species coexistence and decreased the stability of the bacterial communities.

There is a growing interest in understand ing the roles of evolutionary processes in driving species coexistence [12]. An experimental study by Scheuerl et al. [9] demonstrated that bacterial species (obtained from the rainwater in tree holes) had greater evolutionary responses to environmental changes (low $\mathrm{pH}$ ) when the bacterial communities had a low diversity, presumably owing to weaker competitive interactions. These authors show that strong competitive interactions, as found in the diverse bacterial communities, limit 


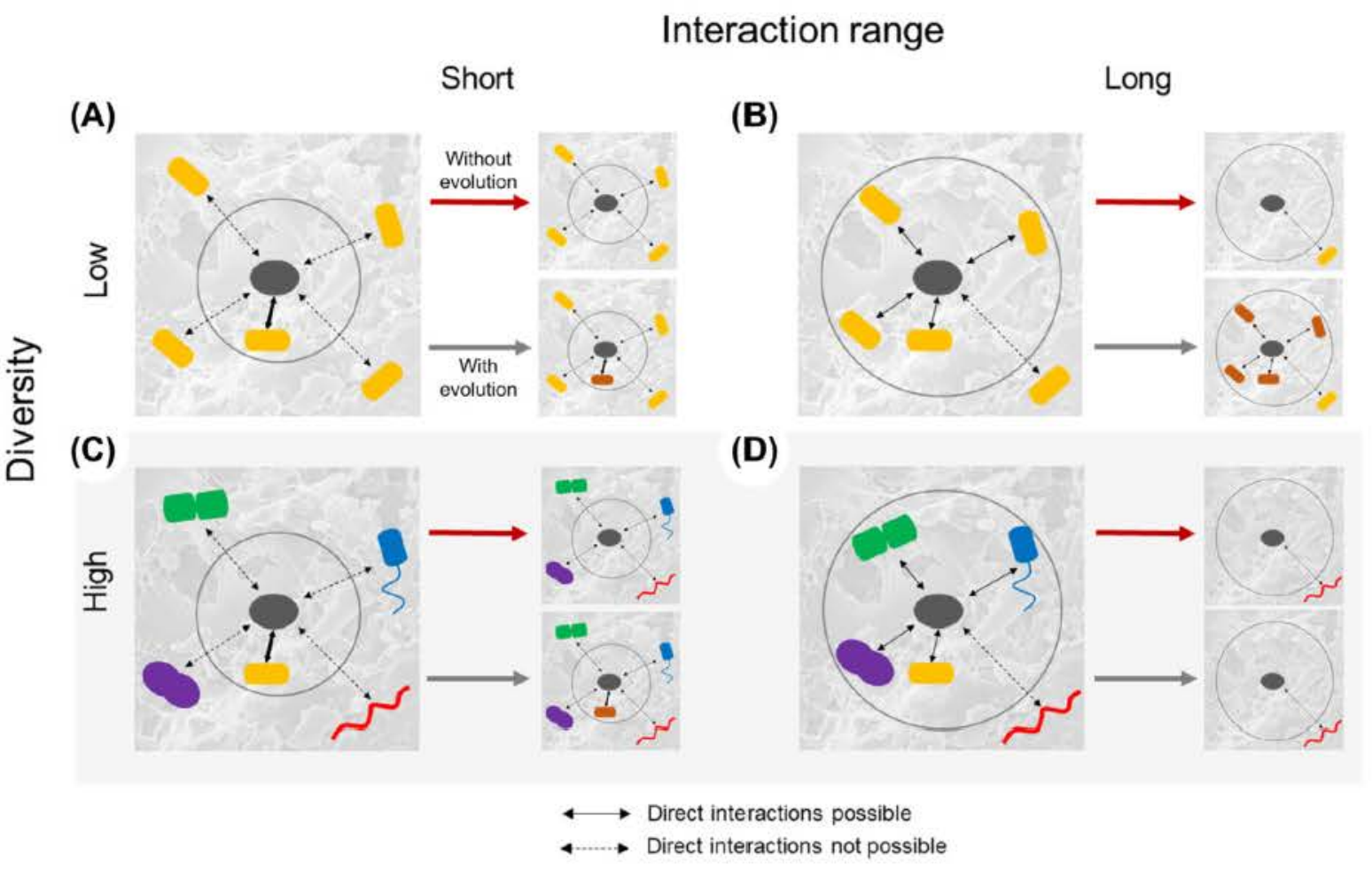

Trends in Microbiology

Figure 1. Some General Rules and Outcomes of Bacterial Interactions. Interaction range (short and long), interaction strength (weak and strong), and community context (low and high diversity) affect bacterial coexistence and evolution. We discuss several possibilities for bacterial coexistence using an example of a focal bacterial species (gray filled) and its interaction with neighboring bacterial species. For simplicity, we focus on competitive interactions, but the framework can be adapted to include both competitive and facilitative interactions. (A) Low-diversity, short-interaction range. In a low-diversity scenario, when the gray bacterial species has short interaction range (indicated by the gray circle) and interaction strength (indicated by the thicker double-sided arrows), it excludes its nearest neighbors, like the yellow bacterial species, by its ability to modify the environment $(e . g$. ., the $\mathrm{pH})$. However, when considering evolution, such competitive interactions could also lead to diversification (indicated by the emergence of the orange bacteria) when the yellow bacteria can adapt to the new environment modified by the gray bacteria. (B) Low-diversity, long-interaction range. When the same gray bacterial species has a longer interaction range it excludes more yellow bacteria in the absence of evolution, whereas a greater diversification of the yellow bacteria takes place in the presence of evolution. (C) High-diversity, short-interaction range. In this scenario, we illustrate the example of five bacterial species living next to the gray bacteria, but only one of them (the yellow one) falls within the short interaction range. The results are identical to the outcomes shown in scenario (A) despite the difference in species diversity. (D) High-diversity, long-interaction range. When the same gray bacterial species has a longer interaction range it excludes the four neighboring species within its interaction range in the absence of evolution. When considering evolution, Scheuerl et al. [9] showed that evolution is constrained when multiple species are interacting (presumably owing to strong interaction strength - not shown in the figure), implying that evolution might not be rapid enough to prevent exclusion of the four neighboring species.

the capacity of bacterial species to evolve, thereby highlighting the importance of 'community context' as a key predictor of bacterial evolutionary dynamics. Taken together, these three recent independent studies [7-9] have provided us with potential general rules on how bacteria interact, and further highlight the often overlooked implications of bacterial interactions for evolutionary dynamics (Figure 1).

\section{Outlook}

We suggest that interaction range, interaction strength, and community context should be considered simultaneously for a better understanding of bacterial interactions. In particular, considering evolution will enhance our ability to predict variations in a bacterial community in a given environment (some scenarios are shown in Figure 1). Microbial ecologists have con- sistently emphasized the importance of making microbial ecology a predictive science rather than just a descriptive science as most research in microbial ecology still continues to be on microbial inventories in various environments $[3,6]$. We believe that, to make microbial ecology more predictive, we need to integrate general rules of microbial interactions to study microbial community diversity, functioning, and 
evolution. The rules highlighted by the three studies discussed earlier [7-9] are an important step towards this goal. Future studies can further explore how these rules may affect each other (Figure 1) - for instance, how the interaction range of bacteria and their ability to modify the environment relate to each other. Moreover, it will be important to test the significance of interaction range, interaction strength, and community context for regulating bacterial community structure in the context of bacterial interactions with other microorganisms (e.g., fungi) and their predators (e.g., protists), and also in the context of different and novel environments, such as those created by climate warming and drought.

\section{Acknowledgments}

We thank two anonymous reviewers for heipful comments. Z.Z. acknowledges funding from the
China Scholarship Council (201606100049). M.P.T acknowledges funding from the German Research Foundation (DFG, TH 2307/1-1, 2-1).

Ecology, Department of Biology. University of Konstanz. 8464, Konstanz, Germany

Zhejiang Provincial Key Laboratory of Plant Evolutionay Ecology and Conservation, Taizhou University, Taizhou 318000. China

Aquatic Ecology and Evolution, Department of Biology. University of Konstanz, 78464, Konstarz, Germany

References

1. Gause, G.F. (1934) Experimental anabsis of Vito Voltern's mathematical theory of the strugge for existence. Science 79, 16-17

2. Kassen, R. of al (2000) Diversity peaks at intermediate productivity in a taboratory microcosm. Nature 406. 508-512

3. Lirse, AEF, et al $(2008$, Pues of engagement interspecies interactions that regiate microbial communties. Amu. Alev. Mcrotiol 62, 375-401
Koncola, A, (2009) What is micrcbial community ecology. ISME J. 3. 1223-1230

5. Faust, K and Raes, J (2012) Microbeal interactions: From networks to models. Nat. Fiev. Microbiol. 10, 538-550

6. Proseer, J.t. and Martiny, J.B.H. (2020) Conceptua chalenges in microbial community ecology. Philos. Trans. R. Soc. B Biol. Sol. 375, 20190241

7. Dal $\mathrm{CO}, \mathrm{A}$ ef al. (2020) Short-range interactions govem the dynamics and functions of microbial communities. Nat. Ecol. Evol. 4. 366-375

8. Ratzke, C. et at. (2020) Strength of species interactions datemines bibdversity and stability in microbial communities Nat Eood. Evol. 4, 376-383

9. Scheverl, T. of al. (2020) Bacterial adaptation is constrained in complex communities. Nat. Commun. 11 , 754

10. Nadell, C.D. et al. (2016) Spatial structure, cooperation and competition in bioflims. Nat. Rev. Microbiol. 14 $589-600$

11. Goldtord, JE et al, (2018) Emergent simpicity in microbie community assembiy. Science 361, 469-474

12. Turootte, MM and Levine, J.M. (2016) Phenotypic plasticity and species coesistences. Trends Ecol. Eval. 31, $803-813$ 